\title{
Innovations in the psychosocial treatment of youth with anxiety disorders: implications for a stepped care approach
}

\author{
Thomas H Ollendick, ${ }^{1}$ Lars-Göran Öst, ${ }^{2}$ Lara J Farrell ${ }^{3}$
}

${ }^{1}$ Department of Psychology, Virginia Tech, Blacksburg, Virginia, USA; ${ }^{2}$ Department of Psychology, Stockholm University, Stockholm, Sweden; ${ }^{3}$ School of Applied Psychology, Griffith University, Southport, Queensland, Australia

Correspondence to Dr Thomas H Ollendick, Department of Psychology, Virginia Tech, Blacksburg, VA 24061, USA; tho@vt.edu

\section{ABSTRACT}

Anxiety disorders are highly prevalent among children and adolescents and frequently result in impairments across multiple domains of life. While psychosocial interventions, namely cognitive-behavioural therapy (CBT), have been found to be highly effective in treating these conditions, significant numbers of youth simply do not have access to these evidence-based interventions, and of those who do, a substantial proportion (up to 40\%) fail to achieve remission. Thus, there is a pressing need for innovation in both the delivery of evidence-based treatments and efforts to enhance treatment outcomes for those who do not respond to standard care. This paper reviews current innovations attempting to address these issues, including evidence for brief, low-intensity approaches to treatment; internet delivered CBT and brief, high-intensity CBT. Moreover, we propose a model of stepped care delivery of evidence-based mental health interventions for children and youth with anxiety. In general, a stepped care approach begins with a lower intensity, evidence-based treatment that entails minimal therapist involvement (ie, brief, low-intensity self-help or internet delivered CBT) and then proceeds to more intensive treatments with greater therapist involvement (ie, brief high-intensity CBT), but only for those individuals who show a poor response at each step along the way. Future research is needed in order to evaluate such a model, and importantly, to identify predictors and moderators of response at each step, in order to inform an evidence-based, fully-integrated stepped care approach to service delivery.

\section{INTRODUCTION}

Anxiety disorders are common in childhood and adolescence, with up to $10 \%$ of children and $20 \%$ of adolescents meeting criteria for an anxiety disorder at any one point in time. ${ }^{1}$ Moreover, findings from the National Comorbidity Survey Replication-Adolescent Supplement ${ }^{2}$ conducted in the USA revealed that $31.9 \%$ of the adolescents met a lifetime prevalence of any anxiety disorder. In the past 20-25 years, the efficacy of cognitive-behavioural therapy (CBT) for the treatment of these disorders in youth has been well documented (see 3, 4 for reviews). For the most part, this evidence has been accrued from 'standard' CBT treatments typically consisting of 9-18 weekly sessions delivered on an outpatient basis in either individual or group format and with varying levels of parental involvement. Although effective for most youth, between $25 \%$ and $40 \%$ are not diagnosis free following treatment. ${ }^{3} 4$ Furthermore, a recent systematic review of long-term outcome studies spanning 2-19 years following treatment found that approximately $30 \%$ of children failed to achieve remission over time. ${ }^{5}$ Thus, anxiety disorders continue to result in considerable impairment and distress for a significant number of youth even following evidencebased psychosocial treatments.

In partial response to these less than optimal outcomes, the use of other interventions as supplements to 'standard' CBT treatments or as standalone treatments for child and adolescent anxiety disorders has evolved. In their recent book on improving access to care and enhancing treatment outcomes for the paediatric anxiety disorders, Farrell et a ${ }^{6}$ explored a number of developments each of which utilised the two primary components of standard CBT: cognitive challenges and graduated exposure.

\section{METHODS}

Here, we highlight three of these developments: (1) brief, low-intensity interventions; (2) internet and computer-based approaches and (3) brief, high-intensity interventions. Following brief review of these interventions, we propose a stepped care approach to service delivery for anxious youth. In general, stepped care models aim to provide effective yet cost-efficient interventions starting with evidencebased, low-intensity treatments with minimal therapist involvement and proceeding to more intensive treatments with greater therapist involvement but only for those individuals who show a poor response. ${ }^{7}$

\section{PRESENTATION}

\section{Brief, low-intensity interventions}

Within a stepped care approach, these brief interventions are designed to reach more families, to be more efficient and to be most cost-effective. Bennett-Levy and Farrand ${ }^{8}$ refer to the development of these brief, low-intensity interventions as constituting a paradigm shift-one that constitutes a revolution in the delivery of mental healthcare. A variety of such interventions exist, ranging from self-help books, 30 min advice clinics and brief interventions prescribed by family physicians and mental health professionals in primary care settings. Illustrating this latter approach, Strosahl and Robinson ${ }^{9}$ recently reviewed the evidence associated with brief 30 min interventions in primary care settings for a variety of mental health problems in adults; however, as noted by them, limited evidence for their efficacy with anxious youth exists at this time. The first randomised controlled trial of a parent-delivered self-help for child anxiety with minimal therapist guidance was undertaken by Rapee and colleagues. ${ }^{10}$ This trial examined the relative efficacy of a self-help book written for parents ('Helping Your Anxious Child'), to a face-to-face, clinic-based CBT programme and a waitlist control condition. Children with generalised anxiety disorder, separation anxiety disorder, social anxiety disorder and panic disorder were enrolled. The self-help book alone resulted in $26 \%$ recovery rate, compared with $61 \%$ for those in the face-to-face intervention and $7 \%$ for those in the waitlist. Importantly, Lyneham and Rapee ${ }^{11}$ subsequently showed that when the self-help book was supplemented with direct support and guidance to the parents from a therapist, the recovery rates increased substantially. Furthermore, Creswell and colleagues ${ }^{12}$ as well as Cobham et al ${ }^{13}$ have recently shown positive outcomes for parent-delivered CBT interventions when supplemented by therapist support. For example, while brief, guided parent-delivered CBT was not superior to brief solution-focused therapy for child anxiety $(n=136,5-12$ years) across four community mental health clinics in the UK, findings did support cost-effectiveness of the brief, guided parent-delivered CBT, suggesting this approach may be an appropriate, effective first-line treatment in National Mental Health Services.

Although low-intensity interventions, such as self-help interventions (without therapist support), may be less effective than face-to-face clinic-based treatments, Bennett-Levy and Farrand ${ }^{8}$ note that these interventions allow for patient choice as some individuals prefer and feel more comfortable with such approaches over longer one-to-one therapy 
sessions as delivered in the standard format and in mental health outpatient clinics. Of course, such approaches also allow greater access to services, especially so in areas that do not have trained professionals readily available; indeed, it is unlikely that there will ever be a sufficiently large mental health workforce to meet the needs of youth and their families in our societies. Finally, Bennett-Levy and Farrand ${ }^{8}$ indicate that CBT, given its evidentiary support and its sound underlying therapeutic principles, is uniquely positioned to be in the foreground of this revolution. Although much work remains to be done, ${ }^{14}$ these approaches, especially when augmented with minimal therapist input (eg, 12), appear promising as a 'first-line' approach to reaching the many youth with anxiety disorders.

\section{Internet and computer-based approaches}

Computer, mobile and internet delivery methods are a second way of increasing access to mental health treatment for children and adolescents. ${ }^{15}$ The majority of children in most Western societies now have access to the internet via a computer or a mobile device and they tend to be highly skilled in the use of such technologies. Moreover, Spence and colleagues ${ }^{15}$ report the internet as being a key source through which adolescents seek information about their mental health concerns. Of course, CBT is also well suited for online and computerised delivery since it is highly structured and typically involves a series of clearly specified therapy components or elements that can be implemented in a systematic and sequential fashion. A relatively large amount of research documents the effectiveness of this approach for adults with anxiety disorders. ${ }^{16} 17$

As noted by Hollis and colleagues ${ }^{18}$ and Spence and colleagues, ${ }^{15}$ a limited number of studies support the efficacy of this approach with children and adolescents. More specifically, the percent of youth free from pretreatment anxiety diagnoses tends to be higher in those studies which include therapist contact during the delivery of the online programmes: the percent free of anxiety diagnosis is between $56 \%$ and $80 \%$ when a therapist actively participates in the treatment, whereas it is around $20 \%-40 \%$ at post-treatment when there is minimal or no therapist involvement in these studies. Although the effects for the minimal therapist involvement were relatively small in these studies, they were significantly greater than that obtained in wait list control conditions in several studies $(<10 \%)$. Moreover, at long-term follow-up, the number of youth free from their pretreatment diagnoses increased for both online conditions. The recent work of Vigerland and colleagues demonstrates both the immediate and lasting effects in an internet-based intervention that targets parents and their anxious children. ${ }^{1920}$ Thus, it appears that internet-delivered interventions, with at least some therapist involvement can result in significant reductions in anxiety both at post-treatment and long-term follow-up. Importantly, internet-delivered interventions have the potential to reach a significant minority of youth who might not have access to standard, weekly face-to-face treatments. ${ }^{21}$ From a stepped care public health perspective, such findings are most important ones. However, as with self-help approaches, it will be necessary to replicate and extend these findings in future research and determine for whom these approaches are most likely to be effective through studies of predictors and moderators of treatment outcome.

\section{Brief, high-intensity interventions}

In general, brief high-intensity approaches have modified more traditional or conventional CBT approaches by reducing either the number of sessions or the time period over which the sessions are delivered. High, intensive CBT treatments, like traditional CBT, is typically delivered faceto-face and in a clinical setting - in contrast to many of the self-help and internet-based interventions. In a sense, and not unlike low-intensity CBT, these approaches also constitute a revolution in delivery of mental health services in that they provide psychotherapy in a non-traditional format outside the typical 'therapy hour' and in fewer than the 9-18 sessions that characterise traditional CBT. ${ }^{22-25}$

These brief interventions, like standard CBT, rely on cognitive change and exposure as critical components of treatment. Based on learning theory, Bouton ${ }^{26}$ early on showed that original feared associations (CS-US pairings) that characterise anxiety can be inhibited by newly formed CS-non-US pairings. Although fear memories are not erased, they are inhibited and 'contextualised' by the newly acquired pairings. Craske and colleagues ${ }^{27}$ suggest that for feared associations to change and for exposure to be maximally effective, treatment should be delivered in sessions that are close in proximity and that involve multiple situations for the new pairings to be formed. Thus, we believe that brief, intensive CBT approaches provide the necessary therapeutic context to maximise exposure therapy outcomes.

In a recent meta-analysis of 23 brief, high-intensity treatments across the anxiety disorders, Öst and Ollendick ${ }^{23}$ documented that these treatments resulted in lower attrition rates (2.3\%) than standard CBT (6.5\%). They also noted the effect sizes (ES) for comparison of these treatments with wait-list (1.47) and placebo (0.91) conditions were significant, whereas the ES for comparison with standard CBT (0.01) was not. Positive outcomes on remission rates were evident at post-treatment and follow-up for these treatments $(54 \% / 64 \%)$ which were equivalent to standard clinic-based CBT (57\%/63\%) and superior to placebo (26\%/35\%) and waitlist control conditions (7\%/9\%). These 23 studies included youth with specific phobia, separation anxiety disorder, social anxiety disorder and panic disorder. From a stepped-care perspective, these brief high-intensity interventions represent an alternative, equally effective but more efficient approach to standard weekly CBT (ie, 9-18 sessions).

\section{Implications for a stepped care approach}

Given the high prevalence of anxiety disorders in youth, the fact that many children and adolescents do not receive the best evidence-based treatments that are available and that a significant minority (up to 40\%) are not diagnosis free following use of these evidence-based interventions, ${ }^{6}$ the need to develop, evaluate and disseminate interventions that are more readily available, accessible, promote adherence and evidencebased is evident. Here, we highlighted three interventions that show considerable promise as standalone treatments for youth with varying levels of anxiety and its disorders. They range from low-intensity self-help strategies (eg, bibliotherapy) to internet/computer-based interventions to brief high-intensity interventions. These interventions vary considerably with the amount of therapist involvement: minimal therapist involvement in the self-help interventions, modest involvement in the internet-based interventions and greatest involvement with the brief intensive CBT interventions. In general, a stepped care approach begins with a low-intensity, evidence-based treatment that entails minimal therapist involvement and then proceeds to more intensive treatments with greater therapist involvement but only for those individuals who show a poor response at each step along the way. ${ }^{6}$

Recently, Rapee et $a{ }^{28}$ published the first RCT of a stepped care treatment for child anxiety. In this trial, 281 youth with anxiety disorders (6-17 years of age) were randomly allocated to receive either empirically validated CBT (standard care) or stepped care involving the following: (1) low-intensity (guided self-help); (2) standard CBT ( $\sim 10$ sessions CBT); and (3) individually tailored treatment $(\sim 10$ sessions tailored CBT). Results revealed no significant differences across the treatment conditions on any outcomes; however, the stepped care model resulted in significantly less therapist time, providing evidence for cost-effectiveness. This RCT provides preliminary empirical support for one approach to stepped care for youth with anxiety disorders and highlights the potential cost benefits and efficiencies of such a model. 


\section{DISCUSSION}

In order for the field to advance, additional research is needed regarding the efficacy of alternate models of stepped care, as well as evidence to inform whom these treatments work best. For example, stepped care models are unlikely to be an efficient model of service delivery for children with severe and comorbid conditions, whereby low-intensity steps may result in undue delays in accessing an adequate dose of intervention. For example, given that pretreatment severity of child anxiety is one known predictor of both short-term and long-term outcomes, ${ }^{5}$ children with subclinical and mild anxiety disorders may be prime candidates for lower intensity interventions, whereas children of moderate severity may be more efficiently and effectively treated by commencing with a brief, but high-intensity intervention in the first step of care. Although such an integrated approach to care has been attempted with adults with anxiety disorders, ${ }^{29}$ it has not yet been attempted in the paediatric anxiety area. Moreover, research is needed to compare the efficacy of low-intensity treatments (step 1), relative to an integrated stepped care model, and treatment-as-usual; in order to determine the efficacy and cost-effectiveness of stepped care, relative to more a single dose of low-intensity treatments.

From a stepped care vantage point, we suggest that a self-helpbased approach (including bibliotherapy with therapist guidance) as well as internet-based CBT (again with therapist assistance) constitute the first step in intervention, at least for those youth who experience mild forms of anxiety disorders. These approaches are widely available and can be supplemented by support from mental health professionals with varying degrees of training. ${ }^{30}$ For those youth who do not respond to this initial step or for youth with more severe presentations, we recommend advancing to or commencing treatment with brief, high-intensity CBT since these abbreviated treatments are equally effective with standard CBT approaches. They are more cost-efficient to the patient and the therapist time commitment is significantly less than that for standard weekly CBT approaches. With this step, another subset of youth is likely to respond. For the remainder of youth who are non-responsive at this step, we propose a second course of brief, high-intensity CBT or a trial of standard CBT. However, we must once again note that although each of these interventions has been shown to be effective for children with anxiety disorders, their utility within a stepped care framework such as we propose here has not yet been empirically tested.

Finally, for those youth who still do not respond, augmentation of CBT with a pharmacologic agent might be considered. A number of trials using the selective serotonin reuptake inhibitors (SSRIs) have shown the superiority of this agent over placebo in children with separation, social and generalised anxiety disorders. These preliminary studies culminated in the Child/Adolescent Anxiety Multimodal Study (CAMS). ${ }^{31}$ CAMS was the first large, multicentre, comparative efficacy study of 488 youth suffering from separation, social and generalised anxiety disorders and evaluated the efficacy of not only the SSRI, sertraline, but also CBT, their combination and a pill placebo control condition. This important study showed that sertraline was equivalent to standard CBT with both treatments superior to a placebo control condition. It also showed that the combination of CBT with sertraline was far superior to either treatment alone, resulting in near $80 \%$ recovery rate at post-treatment. However, this difference was no longer present at 6 months or 6-year follow-up, as all treatments were equally effective. More recently, the serotonin norepinephrine reuptake inhibitor (SNRI) duloxetine was shown to be more effective than a pill placebo in children and adolescents with generalised anxiety in a randomised control trial. ${ }^{32}$ Unfortunately, duloxetine was not compared with CBT in this trial nor was it explored as an augmentation agent to boost the effects of CBT, potentially resulting in even greater outcomes than when used alone. While considerable evidence supports the use of SSRIs over placebos for paediatric anxiety disorders, at least this one study by Strawn and colleagues ${ }^{32}$ demonstrates the efficacy of a SNRI, providing an additional avenue to explore if the SSRIs are not shown to be effective augmentation strategies. We exercise additional caution here however as a variety of symptoms including dizziness, lightheadedness, excessive sweating, irritability, dysphoria and insomnia have been reported when these medications are both administered and discontinued. In addition, assuming the SSRI or SNRI is effective, Avari and colleagues ${ }^{33}$ have recently recommended that the medication be continued for up to 1 year after remission to ensure that the child has the best chance to come off the medication without a return of symptoms. To us, the negative side effects and the necessity of prolonged administration of the drug even following remission suggest that these pharmacological agents be used only after at least two adequate courses of CBT have been attempted and only when they are used in combination with CBT to be maximally effective.

\section{CONCLUSIONS}

In sum, we propose that more youth can be reached within a stepped care approach that includes evidence-based interventions at various levels of care. Stepped care approaches provide greater flexibility laccommodating for therapist and patient preferences), more efficient models of care and the possibility that more patients will achieve remission. Although the future of stepped care for youth with anxiety is drawing near, much research is desperately needed to establish the predictors and moderators of treatment response and remission rates at each level of care. As noted by Bower and Gilbody, ${ }^{7}$ the potential clinical and economic benefits of stepped care are likely dependent on underlying assumptions of equivalence in terms of clinical outcomes at various levels of care, demonstrated efficiency in terms of resource use and costs, and the acceptability of what they refer to as 'minimal interventions' to both patients and therapists. Furthermore, an empirically supported implementation framework must be developed to fully evaluate this stepped care approach.

\section{Competing interests None declared.}

Provenance and peer review Not commissioned; externally peer reviewed.

(C) Author(s) (or their employer(s)) 2018. No commercial re-use. See rights and permissions. Published by BMJ.

doi:10.1136/eb-2018-102892

Received 31 January 2018; Revised 4 June 2018; Accepted 5 June 2018

\section{REFERENCES}

1. Essau CA, Gabbidon J. Epidemiology, comorbidity, and mental health services utilization. In: Essau CA, Ollendick TH, eds. The Wiley-Blackwell handbook of the treatment of childhood and adolescent anxiety. Chichester, UK: John Wiley \& Sons Ltd, 2013:23-41.

2. Merikangas KR, He JP, Burstein M, et al. Lifetime prevalence of mental disorders in U.S. adolescents: results from the National Comorbidity Survey Replication--Adolescent Supplement (NCS-A). J Am Acad Child Adolesc Psychiatry 2010;49:980-9.

3. Higa-McMillan CK, Francis SE, Rith-Najarian L, et al. Evidence Base Update: 50 Years of Research on Treatment for Child and Adolescent Anxiety. J Clin Child Adolesc Psychol 2016:45:91-113.

4. James AC, James G, Cowdrey FA, et al. Cognitive behavioural therapy for anxiety disorders in children and adolescents. Cochrane Database Syst Rev 2015:1-85.

5. Gibby BA, Casline EP, Ginsburg GS. Long-Term Outcomes of Youth Treated for an Anxiety Disorder: A Critical Review. Clin Child Fam Psychol Rev 2017;20:201-25.

6. Farrell L, Ollendick TH, Muris P, eds. Innovations in CBT for childhood anxiety, OCD, and PTSD: Improving access \& outcome. Cambridge: Cambridge University Press. In Press.

7. Bower $\mathbf{P}$, Gilbody S. Stepped care in psychological therapies: access, effectiveness and efficiency. British Journal of Psychiatry 2005;186:11-17.

8. Bennett-Levy J, Farrand P. et a/ln: Bennett-Levy J, Richards DA, Farrand P, Christensen H, Griffiths KM, . eds. Low intensity CBT models and conceptual underpinnings. Oxford: Oxford University Press, 2010.

9. Strosahl KD, Robinson PJ. Harnessing the power of brief behavioral interventions: Seven principles of success. Workshop presented at the $51^{\text {st }}$ Annual Convention of the Association of Behavioral and Cognitive Therapies. San Diego, CA 2017.

10. Rapee RM, Abbott MJ, Lyneham HJ. Bibliotherapy for children with anxiety disorders using written materials for parents: A randomized controlled trial. J Consult Clin Psychol 2006; 74:436-44. 
11. Lyneham HJ, Rapee RM. Evaluation of therapist-supported parent-implemented CBT for anxiety disorders in rural children. Behav Res Ther 2006;44:1287-300.

12. Creswell C, Violato M, Fairbanks H, et al. Clinical outcomes and cost-effectiveness of brief guided parent-delivered cognitive behavioural therapy and solution-focused brief therapy for treatment of childhood anxiety disorders: a randomised controlled trial. Lancet Psychiatry 2017:4:529-39.

13. Cobham VE, Filus A, Sanders MR. Working with parents to treat anxiety-disordered children: A proof of concept RCT evaluating Fear-less Triple P. Behav Res Ther 2017:95:128-38.

14. McLellan L, Fitzpatrick S, Schniering C, et al. Self-help treatment of childhood anxiety disorders. In: Farrell L, Ollendick TH, Muris P, eds. Innovations in CBT for childhood anxiety, OCD, and PTSD: Improving access \& outcome. Cambridge: Cambridge University Press.

15. Spence SH, March S, Donavan C. New technologies to deliver CBT: Computer and web-based programs, mobile applications and virtual reality. In: Farrell L, Ollendick TH, Muris P, eds. Innovations in CBT for childhood anxiety, OCD, and PTSD: Improving access \& outcome. Cambridge: Cambridge University Press.

16. Adelman CB, Panza KE, Bartley CA, et al. A meta-analysis of computerized cognitivebehavioral therapy for the treatment of DSM-5 anxiety disorders. J Clin Psychiatry 2014;75:e695-704.

17. Craske MG, Rose RD, Lang A, et al. Computer-assisted delivery of CBT for anxiety disorders in primary care settings. Depress Anxiety 2009;26:235-42.

18. Hollis C, Falconer CJ, Martin JL, et al. Annual Research Review: Digital health interventions for children and young people with mental health problems - a systematic and meta-review. J Child Psychol Psychiatry 2017;58:474-503.

19. Vigerland S, Ljótsson B, Thulin U, et al. Internet-delivered cognitive behavioural therapy for children with anxiety disorders: A randomised controlled trial. Behav Res Ther 2016;76:47-56.

20. Vigerland S, Serlachius E, Thulin U, et al. Long-term outcomes and predictors of internet-delivered cognitive behavioral therapy for childhood anxiety disorders. Behav Res Ther 2017;90:67-75.

21. Rooksby M, Elouafkaoui P, Humphris G, et al. Internet-assisted delivery of cognitive behavioural therapy (CBT) for childhood anxiety: systematic review and meta-analysis J Anxiety Disord 2015;29:83-92.
22 Ollendick TH. Brief, high intensity interventions with children and adolescents with anxiety disorders: Introduction and commentary. Psychopathol Rev 2014;1:169-74.

23 Öst LG, Ollendick TH. Brief, intensive and concentrated cognitive behavioral treatments for anxiety disorders in children: A systematic review and meta-analysis. Behav Res Ther 2017;97:134-45.

24. Pincus DB, Hardway C. Brief intensive treatments. In: Farrell L, Ollendick TH, Muris $P$, eds. Innovations in CBT for childhood anxiety, OCD, and PTSD: Improving access \& outcome. Cambridge: Cambridge University Pressln press.

25. Wright JH, Sudak DM, Turkington D, et al; High yield cognitive-behavior therapy for brief sessions: An illustrated guide. Washington, D.C.: American Psychiatric Press, 2010.

26. Bouton ME. Context, time, and memory retrieval in the interference paradigms of Pavlovian learning. Psychol Bull 1993;114:80-99.

27. Craske MG, Liao B, Brown L, et al. Role of inhibition in exposure therapy. J Exp Psychopathol 2012;3:jep.026511-345.

28. Rapee RM, Lyneham HJ, Wuthrich V, et al. Comparison of Stepped Care Delivery Against a Single, Empirically Validated Cognitive-Behavioral Therapy Program for Youth With Anxiety: A Randomized Clinical Trial. J Am Acad Child Adolesc Psychiatry 2017; 56:841-8.

29. Nordgreen T, Haug T, Öst LG, et al. Stepped Care Versus Direct Face-to-Face Cognitive Behavior Therapy for Social Anxiety Disorder and Panic Disorder: A Randomized Effectiveness Trial. Behav Ther 2016;47:166-83

30. Salloum A. Minimal therapist-assisted cognitive-behavioral therapy interventions in stepped care for childhood anxiety. Prof Psychol 2010;41:41-7

31. Walkup JT, Albano AM, Piacentini J, et al. Cognitive behavioral therapy, sertraline, or a combination in childhood anxiety. N Engl J Med 2008;359:2753-66.

32. Strawn JR, Prakash A, Zhang Q, et al. A randomized, placebo-controlled study of duloxetine for the treatment of children and adolescents with generalized anxiety disorder. J Am Acad Child Adolesc Psychiatry 2015;54:283-93.

33. Avari JM, Cohen MG, Hatziergati D, et al. Pharmacologic-enhanced approaches for the anxiety disorders. In: Farrell L, Ollendick TH, Muris P, eds. Innovations in CBT for childhood anxiety, OCD, and PTSD: Improving access \& outcome. Cambridge: Cambridge University Press. 